\title{
Equações de perfil do tronco avaliados para Corymbia citriodora Hill \& Johnson
}

\section{Trunk profile equations evaluated for Corymbia citriodora Hill \& Johnson}

\author{
Valdir Carlos Lima de Andrade \\ Universidade Federal do Tocantins \\ E-mail: vclandrade@uft.edu.br \\ OrcID: https://orcid.org/0000-0002-5559-9124
}

Brenno Cunha Freitas

Universidade Federal do Tocantins

E-mail: brennofreitas_10@hotmail.com

OrcID: https://orcid.org/0000-0002-0606-8917

David Lucas Camargo Vieira Terra

Universidade Federal do Tocantins

E-mail: davidlcvt7@hotmail.com

OrcID: https://orcid.org/0000-0002-5762-0804

Resumo: Equações de perfil do tronco das árvores viabilizam quantificar diferentes usos que se possam destinar a produção madeireira de um determinado povoamento florestal. São advindas do ajuste de modelos de taper, referenciados como: modelos de afilamento, adelgaçamento, forma e, ou de perfil do tronco ou do fuste. Como não se encontram estudos sobre o emprego de equações de perfil do tronco em plantios brasileiros com Corymbia citriodora, esse trabalho objetivou verificar o ajuste de uma grande quantidade de modelos para esta importante espécie florestal. Foram utilizadas 35 árvores cubadas com medições diretas do diâmetro do tronco nas posições: $0,2 \mathrm{~m}, 0,4 \mathrm{~m}, 0,7 \mathrm{~m}, 1,3 \mathrm{~m}, 2,7 \mathrm{~m}$ e a cada 2 metros até um valor aproximado de $1,0 \mathrm{~cm}$ com casca. Como critérios estatísticos de avaliação das equações de perfil do tronco geradas para C. citriodora, foram considerados: coeficiente de determinação ajustado, erro padrão da estimativa, distribuição gráfica dos resíduos, média dos desvios absolutos, desvio padrão das diferenças, soma dos quadrados dos resíduos relativos, média dos desvios percentuais, raiz quadrada do erro médio, viés e coeficiente de correlação linear múltipla. Concluiu-se que, para descrever o perfil do tronco de $C$. citriodora, as equações geradas por modelos muito difundidos no Brasil apresentaram desempenho insatisfatório e que se destacaram seis modelos ainda pouco testados ou sem aplicação no país.

Palavras-chave: Validação cruzada. Taper. Multiprodutos madeireiros.

\begin{abstract}
Profile equations of the trunk of the trees make it possible to quantify different wood uses that can be used in a specific forest stand. They come from the adjustment of taper models, referred to as: tapering, thinning, shape and, or profile of the trunk or shaft. Since there are no studies on the use of trunk profile equations in Brazilian plantations with Corymbia citriodora, this study aimed to verify the fit of a large number of models for this important forest species. 35 cubed trees were used with direct measurements of the diameter of the trunk in the positions: $0.2 \mathrm{~m}, 0.4 \mathrm{~m}, 0.7 \mathrm{~m}, 1.3 \mathrm{~m}, 2.7 \mathrm{~m}$ and every 2 meters up to an approximate value of $1.0 \mathrm{~cm}$ shelled. As statistical criteria for evaluating the trunk profile equations generated for $C$. citriodora, the following were considered: adjusted determination coefficient, standard error of the estimate, graphical distribution of the residues, mean of the absolute deviations, standard deviation of the differences, sum of the squares of the residues relative, mean of the percentage deviations, square root of the mean error, bias and multiple linear correlation coefficient. It was concluded that, to describe the profile of the trunk of $C$. citriodora, the equations generated by models that are very widespread in Brazil presented an unsatisfactory performance and that six models that are still under-tested or without application in the country stand out.
\end{abstract}

Keywords: Cross-validation. Taper. Wood Products. 


\section{Introdução}

Os primeiros trabalhos brasileiros sobre o uso de equações de perfil do tronco datam dos anos 70 , quando houve o prelúdio da necessidade em se quantificar os diferentes usos da madeira. Tais equações, por permitirem descrever o perfil longitudinal do tronco das árvores em pé, geram a predição de três informações básicas: o diâmetro em qualquer posição do fuste, a altura em que ocorre um determinado diâmetro comercial e o volume entre dois pontos quaisquer. Informações estas, que permitem uma decisão melhor balizada quanto ao uso da madeira para laminação, serraria, celulose, energia, mourões, postes, construção civil e, ou, dentre muitos outros produtos madeireiros que um mesmo povoamento florestal pode produzir (Andrade, 2014).

Scolforo (1993) ressalta situações onde se exige à aplicação de equações de perfil do tronco devido a estes permitir definir o potencial que uma árvore tem quanto ao seu uso, uma vez que a madeira é o componente de maior importância em determinado empreendimento florestal. Além disso, conforme Fischer et al. (2001), o emprego de equações de perfil do tronco viabiliza também a avaliação do uso da madeira quanto aos aspectos biológicos, silviculturais e econômicos, bem como, a resposta às práticas de manejo adotadas.

Neste sentindo, os estudos brasileiros sobre equações de perfil do tronco de árvores deram maior enfoque em plantios com eucalipto (Ribeiro \& Andrade, 2016; Azevedo et al., 2017; Cerqueira et al., 2018; Souza et al., 2018; Farias, Marcos \& Natalia, 2019); com pinus (Fischer et al., 2001); com outras espécies (Muller et al., 2014). Há, ainda, que se reportar aos trabalhos feitos em floresta nativa (Lanssanova et al., 2013; Andrade et al., 2016). Desses trabalhos, se observa a ausência de estudos brasileiros sobre o perfil do tronco de Corymbia citriodora Hill \& Johnson (C. citriodora), espécie esta que tem uma grande importância no uso madeireiro para serraria, mourões, madeira tratada e produção de carvão vegetal.

Diante do exposto e, dada à importância da madeira de C. citriodora para diferentes usos, esse trabalho objetivou avaliar 34 equações de perfil do tronco para esta espécie.

\section{Material e Métodos}

\section{Coleta de dados e modelos de afilamento avaliados}

Os dados utilizados neste estudo foram coletados em um plantio seminal de $C$. citriodora, com idade de 55 meses e formado no espaçamento inicial de $3 \times 2 \mathrm{~m}$. Sua localização é nas proximidades do município de Gurupi, região sul do Estado do Tocantins, onde o clima é do tipo Aw, tropical com estação seca, temperatura média em torno de 28 a $30^{\circ} \mathrm{C}$ e média pluviométrica em torno de 1.000 a $1.200 \mathrm{~mm} / a n o$ entre outubro e maio (Instituto Nacional de Meteorologia [INMET], 2016).

No plantio amostrado, foram abatidas e cubadas 35 árvores distribuídas em sete classes diamétricas com número igual de indivíduos por classe. As medições ao longo do tronco foram feitas nas posições: 0,2 m, 0,4 m, $0,7 \mathrm{~m}, 1,3 \mathrm{~m}, 2,7 \mathrm{~m}$ e a cada 2 metros até um valor aproximado de $1 \mathrm{~cm}$ com casca. Após a cubagem, que resultou em 283 posições medidas longo do tronco, foram geradas 34 equações de perfil do tronco a partir do ajuste dos seguintes modelos estatísticos:

Baldwin e Feduccia (1991):

$\left(\frac{\mathrm{di}}{\mathrm{d}}\right)=\beta 1+\beta 2 \operatorname{Ln}\left\{1-(\mathrm{z})^{(1 / m)}\left[1-\exp \left(-\frac{\beta 1}{\beta 2}\right)\right]+\varepsilon\right\}$

Demaerschalk (1973):

$\left(\frac{d i}{d}\right)=(10)^{\beta 0}(d)^{(\beta 1-1)}(L)^{\beta 2}(h)^{\beta 3}+\varepsilon$

Kozak et al. I (1969):

$\left(\frac{\mathrm{di}}{\mathrm{d}}\right)^{2}=\beta 0+\beta 1\left(\frac{\mathrm{hi}}{\mathrm{h}-1,3}\right)+\beta 2\left(\frac{\mathrm{hi}^{2}}{(\mathrm{~h}-1,3)^{2}}\right)+\varepsilon$

Kozak (1988): 
$(d i)=\beta 0(d)^{\beta 1} \beta 2^{(d)}\left[\frac{(1-\sqrt{z})}{(1-\sqrt{P})}\right]^{\left[\beta 3(z)^{2}+\beta 4 \operatorname{Ln}(z+0,001)+\beta 5 \sqrt{z}+\beta 6 \exp (z)+\beta 7\left(\frac{d}{h}\right)\right]}$

Kozak (2004):

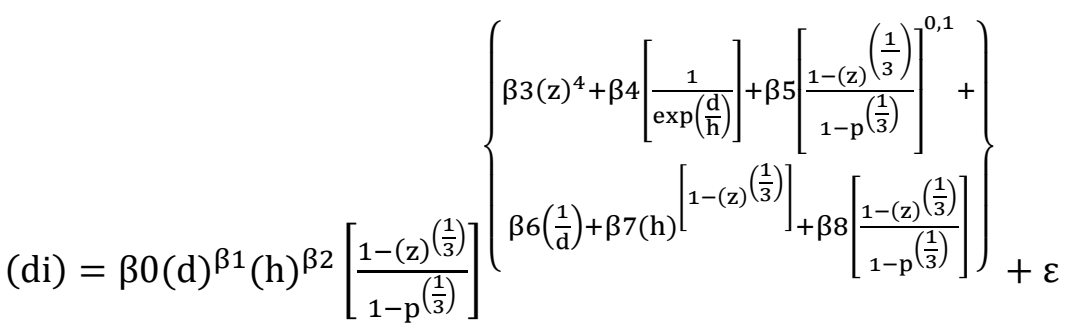

Ormerod I (1973):

$\left(\frac{d i}{d}\right)=\beta 0(X)^{\beta 1}+\varepsilon$

Schöepfer I (1966):

$(d i)=\beta 0+\beta 1(d)+\beta 2(z)(d)+\beta 3(z)^{2}(d)+\beta 4(z)^{3}(d)+\beta 5(z)^{4}(d)+\beta 6(z)^{5}(d)+\varepsilon$

Hradetzky (1976):

$\left(\frac{d i}{d}\right)=\beta 0+\beta 1(z)^{p_{1}}+\beta 2(z)^{p_{2}}+\ldots+\beta n(z)^{p_{n}}+\varepsilon$

Garay (1979):

$\left(\frac{\mathrm{di}}{\mathrm{d}}\right)^{2}=\beta 1\left[1+\beta 2 \operatorname{Ln}\left(1-\beta 3(\mathrm{z})^{\beta 4}\right)\right]+\varepsilon$

Biging (1984):

$\left(\frac{\mathrm{di}}{\mathrm{d}}\right)=\beta 1+\beta 2 \operatorname{Ln}\left[1-(\mathrm{z})^{\frac{1}{3}}\right]\left(1-\mathrm{e}^{\frac{-\beta 1}{\beta 2}}\right)+\varepsilon$

Garcia et al. (1993):

$\left(\frac{\mathrm{di}}{\mathrm{d}}\right)=\sqrt{\beta 1-\beta 2(\mathrm{z})^{0,5}+\beta 3(\mathrm{z})+\varepsilon}$

Polinômio do $3^{\circ} \mathrm{Grau}$ :

$\left(\frac{\mathrm{di}}{\mathrm{d}}\right)=\left[\beta 0+\beta 1(\mathrm{z})+\beta 2(\mathrm{z})^{2}+\beta 3(\mathrm{z})^{3}\right]+\varepsilon$

Guimarães e Leite (1992):

$\left(\frac{d i}{d}\right)=\{\exp [1-\exp (1,3-h i)]\}^{\beta 1}(x)^{\beta 2}+\varepsilon$

Newhan (1988):

$\left(\frac{d i}{d}\right)=\left(\frac{L}{h-1,3}\right)^{\left\{1 /\left[\beta 0+\beta 1\left(\frac{L}{h-1,3}\right)^{6}+\beta 2\left(\frac{d}{h}\right)+\beta 3\left(\frac{L}{h-1,3}\right)^{2}\left(\frac{d}{h}\right)\right]\right\}}+\varepsilon$

Muhairwe I (1999):

$(\mathrm{di})=\beta 1(\mathrm{~d})^{\beta 2} \beta_{3}^{(\mathrm{d})}(1-\sqrt{\mathrm{z}})^{\left[\beta 4(\mathrm{z})^{2}+\beta 5\left(\frac{1}{\mathrm{z}}\right)+\beta 6(\mathrm{~d})+\beta 7(\mathrm{~h})+\beta 8\left(\frac{\mathrm{d}}{\mathrm{h}}\right)\right]}+\varepsilon$

Muhairwe II (1999):

$(\mathrm{di})=\beta 1(\mathrm{~d})^{\beta 2}(1-\sqrt{\mathrm{z}})^{\left[\beta 3(\mathrm{z})+\beta 4(\mathrm{z})^{2}+\beta 5\left(\frac{1}{\mathrm{z}}\right)+\beta 6(\mathrm{z})^{3}+\beta 7(\mathrm{~d})+\beta 8\left(\frac{\mathrm{d}}{\mathrm{h}}\right)\right]}+\varepsilon$ 
Andrade (2014):

$\left(\frac{\mathrm{di}}{\mathrm{d}}\right)=\left\{1-\left(\frac{\mathrm{hi}-1,3}{\mathrm{~h}-1,3}\right) \exp \left[-\beta 1(1-\mathrm{z})^{\beta 2}\right]\right\}^{\beta 3}+\varepsilon$

Hojer (1903):

$\left(\frac{\mathrm{di}}{\mathrm{d}}\right)=\beta 0 \operatorname{Ln}\left[\frac{\beta 1+(\mathrm{x}-2,5)}{\beta 2}\right]+\varepsilon$

Johnson (1911):

$\left(\frac{d i}{d}\right)^{2}=\beta 1(x)^{1,5}+\beta 2\left(x^{1,5}-x^{3}\right)(d)+\beta 3\left(x^{1,5}-x^{3}\right)(h)+\beta 4\left(x^{1,5}-x^{3}\right)(h / d)+\beta 5\left(x^{1,5}-x^{3}\right)(h)^{0,5}+$ $\beta 6\left(\mathrm{x}^{1,5}-\mathrm{x}^{3}\right)(\mathrm{h})^{2}+\varepsilon$

Bennett e Swindel (1972):

$\left(\frac{d i}{d}\right)=\beta 1(x)+\beta 2\left(\frac{L(h-1,3)}{d}\right)+\beta 3\left(\frac{L(h-1,3) h}{d}\right)+\beta 4\left(\frac{L(h-1,3)(h+h i+1,3)}{d}\right)+\varepsilon$

Sharma e Zhang (2004):

$$
\left(\mathrm{di}^{2} \mathrm{~h}\right)=\beta 1\left(\mathrm{~d}^{2}\right)\left(\frac{\mathrm{h}}{1,3}\right)^{2-\left[\beta 2+\beta 3(\mathrm{z})+\beta 4(\mathrm{z})^{2}\right]}(\mathrm{x})+\varepsilon
$$

Muhairwe (1993):

(di) $\left.=\beta 0(d)^{\beta 1} \beta 2^{(d)}[(1-\sqrt{z})]^{\left[\beta 3(z)^{2}+\beta 4 \operatorname{Ln}(z+0,001)+\beta 5 \sqrt{(z)}+\beta 6 \exp (z)+\beta 7\left(\frac{d}{h}\right)\right.}\right]+\varepsilon$

Cervera (1973):

$\left(\frac{d i}{d}\right)=\beta 1+\beta 2(x)+\beta 3(x)^{2}+\beta 4(x)^{3}+\beta 5(x)^{4}+\varepsilon$

Real e Moore (1986):

$\left(\frac{d i}{d}\right)^{2}=(x)^{2}+\beta 1\left(x^{3}-x^{2}\right)+\beta 2\left(x^{8}-x^{2}\right)+\beta 3\left(x^{40}-x^{2}\right)+\varepsilon$

Jiménez et al. (1994):

$\left(\frac{d i}{d}\right)^{2}=\beta 0+\beta 1(z)+\beta 23(z)^{2}+\beta 3(z)^{3}+\beta 4(z)^{4}+\beta 5(z)^{5}+\varepsilon$

Newberry e Burkhart (1986):

$(\mathrm{di})=\beta 1(\mathrm{~d})(\mathrm{x})^{\beta 2}+\varepsilon$

Reed e Green (1984):

$\left(\frac{\mathrm{di}}{\mathrm{d}}\right)^{2}=\beta 1(1-\mathrm{z})^{\beta 2}+\varepsilon$

Bi (2000):

$\left.\left(\frac{\mathrm{di}}{\mathrm{d}}\right)=\left[\frac{\left.\operatorname{Logsi} \frac{\pi}{2} \mathrm{z}\right)}{\log \sin \left(\frac{\pi}{2}\right)\left(\frac{1,3}{\mathrm{~h}}\right)}\right]\right]^{\left[\beta 1+\beta 2 \sin \left(\frac{\pi}{2} \mathrm{z}\right)+\beta 3 \cos \left(\frac{3 \pi}{2} \mathrm{z}\right)+\frac{\beta 4 \sin \left(\frac{\pi}{2} \mathrm{z}\right)}{\mathrm{z}}+\beta 5 \mathrm{~d}+\beta 6 \mathrm{z} \sqrt{\mathrm{d}}+\beta 7 \mathrm{z} \sqrt{\mathrm{h}}\right]}+\varepsilon$

Lee et al. (2003):

$(\mathrm{di})=\beta 1(\mathrm{~d})^{\beta 2}(1-\mathrm{z})^{\beta 3(\mathrm{z})^{2}+\beta 4(\mathrm{z})+\beta 5}+\varepsilon$

Sharma e Parton (2009): 
$\left(\frac{\mathrm{di}}{\mathrm{d}}\right)=\beta 0\left(\frac{\mathrm{h}-\mathrm{h}_{\mathrm{i}}}{\mathrm{h}-1,37}\right)\left(\frac{\mathrm{h}}{1,37}\right)^{\beta 1+\beta 2(\mathrm{z})+\beta 3(\mathrm{z})^{2}}+\varepsilon$

Demaerschalk (1972):

$\left(\frac{\mathrm{di}}{\mathrm{d}}\right)^{2}=(10)^{2 \beta 0}(\mathrm{~d})^{(2 \beta 1-2)}(\mathrm{L})^{2 \beta 2}(\mathrm{~h})^{2 \beta 3}+\varepsilon$

Kozak et al. II (1969):

$\left(\frac{d i}{d}\right)^{2}=\beta 0+\beta 1(z)+\beta 2(z)^{2}+\varepsilon$

Ormerod II (1973):

$\left(\frac{d i}{d}\right)^{2}=(X)^{2 \beta 1}+\varepsilon$

Schöepfer II (1966):

$\left(\frac{d i}{d}\right)=\beta 0+\beta 1(z)+\beta 2(z)^{2}+\beta 3(z)^{3}+\beta 4(z)^{4}+\beta 5(z)^{5}+\varepsilon$

Em que: di=diâmetro medido ao longo do tronco $(\mathrm{cm}), d=D A P(\mathrm{~cm}), \mathrm{h}=$ altura total $(\mathrm{m})$, hi=altura referente a di $(\mathrm{m}), \mathrm{Ln}=$ Logaritmo neperiano, $\mathrm{x}=\left(\frac{\mathrm{h}-\mathrm{hi}}{\mathrm{h}-1,3}\right), \mathrm{L}=(\mathrm{h}-\mathrm{hi}), \mathrm{z}=\left(\frac{h i}{\mathrm{~h}}\right), \beta 0$ à $\beta 8=$ coeficientes de regressão à serem estimados e $\varepsilon=$ erro aleatório do modelo.

Ressalta-se que os 34 modelos de perfil do tronco foram obtidos a partir dos trabalhos de Môra (2015), Andrade (2014), Cerqueira et al. (2018) e Souza et al. (2018) onde se encontram as referências bibliográficas de tais modelos avaliados. Salienta-se sobre os modelos 5 e 19 a 30, que ainda não foram testados em povoamentos florestais brasileiros, sendo estes obtidos a partir do trabalho feito por Cellini et al. (2002).

\section{Critérios estatísticos}

$\mathrm{Na}$ avaliação de equações de perfil do tronco, encontram-se diferentes estratégias adotadas de análises. Por exemplo, nos trabalhos de Cellini, Pastur, Wabö e Lencinas (2002), Cerqueira et al. (2018), Souza et al. (2018) e Farias et al. (2019), foi adotada a técnica de validação com dados dependentes, que consiste em avaliar equações de afilamento empregando-se os mesmos dados utilizados no ajuste. Já, nos trabalhos de Andrade (2014) e Azevedo et al. (2017), foi adotada a técnica de validação com dados independentes, que consiste em avaliar equações de perfil do tronco empregando-se dados diferentes daqueles utilizados no ajuste, geralmente, sendo de $50 \%$ a $70 \%$ das árvores-amostra utilizadas no ajuste e de $30 \%$ a $50 \%$ reservadas para aplicação das equações geradas. Há, também, a técnica de validação cruzada que consiste em dividir os dados em duas amostras iguais onde as equações geradas pela amostra A são aplicadas nos dados da amostra B e vice-versa, como feito por Batista, Couto e Marquesini (2001).

Apesar de ser possível adotar a validação de equações de perfil do tronco com dados dependentes, preferencialmente, se deve adotar técnicas de validação com dados independentes porque se poderá avaliar o comportamento das equações em um cenário de real emprego das mesmas. No entanto, ao se considerar as 35 árvores-amostra deste trabalho, não foi possível analisar as equações de perfil do tronco geradas utilizandose $60 \%$ das árvores para ajuste e as $40 \%$ restantes para validação, uma vez que em cada classe de diâmetro foram cubadas cinco árvores. Seriam utilizadas três árvores por classe diamétrica no ajuste (21 árvores) e duas na aplicação (14 árvores), o que se entende não ser uma quantidade ideal de árvores-amostra para representar um cenário de aplicação das equações.

Diante disso, visando adotar a técnica da validação cruzada, se adaptou as 283 posições medidas ao longo do tronco das 35 árvores em dois bancos de dados diferentes, sendo um destinado a estimativa das equações e outro utilizado na aplicação destas equações geradas e vice-versa. Assim, para gerar o banco de dados 1, utilizou-se as posições do tronco: $0,2 \mathrm{~m}, 0,7 \mathrm{~m}, 4,7 \mathrm{~m}$ e a cada $4,0 \mathrm{~m}$ até a última posição de medição do diâmetro. Já, para gerar o banco de dados 2, utilizou-se as posições do tronco: 0,4 m, 2,7 m e a cada 4,0 m até a última posição de medição do diâmetro diferente do banco de dados 1 . Por este procedimento, se obteve 
dois bancos de dados iguais apenas quanto a $\mathrm{d}$ e $\mathrm{h}$ com um total de 151 e 132 posições do tronco para os bancos de dados 1 e 2 , respectivamente.

Dada a grande quantidade de equações de perfil do tronco geradas, a validação cruzada foi realizada em uma terceira etapa de análise com a aplicação das equações que se sobressaíram dentre as 34 geradas. Assim, em uma primeira etapa de avaliação, foram selecionadas somente as equações que apresentaram uma distribuição de resíduos sendo a mais homogênea e equilibrada possível ao longo do eixo de zero $\mathrm{cm}$ em relação ao diâmetro di. Posteriormente, em uma segunda etapa de avaliação, foram considerados os critérios: erro padrão residual (EPR) e coeficiente de determinação ajustado ( $\left.R^{2} a j\right)$, ambos sendo recalculados para a variável di em todas as equações selecionadas na primeira etapa de avaliação (Schneider, Schneider \& Souza, 2009).

Em uma terceira etapa de avaliação, as equações que se sobressaíram na etapa anterior foram analisadas por meio de uma validação cruzada utilizando-se dois bancos de dados diferentes quanto às posições do tronco. Nesta oportunidade, os critérios estatísticos adotados na validação cruzada, além da análise gráfica da distribuição de resíduos, conforme utilizados por Cellini et al. (2002), Andrade (2014), Cerqueira et al. (2018) e Souza et al. (2018), foram: média dos desvios absolutos (MDA), desvio padrão das diferenças (SD), Soma dos quadrados dos resíduos relativos (SQRR), média dos desvios percentuais (MDP), raiz quadrada do erro médio (REQM), viés $(V)$ e coeficiente de correlação múltipla ( $r$ ).

Do mesmo modo ao início das análises anteriores, se descartou as equações de perfil do tronco que apresentaram as piores distribuições de resíduos tanto em um, como nos dois bancos de dados diferentes. Nesse caso, a análise gráfica da distribuição de resíduos, feita no banco de dados 2, foi resultado da aplicação das equações ajustadas pelo banco de dados 1 e vice-versa. Em seguida, conforme utilizado por Lanssanova et al. (2013) e Ribeiro e Andrade (2016), realizou-se uma classificação dos modelos selecionados nesta etapa considerando-se MDA, SD, SQRR, MDP, RQEM, V e r.

Para o fim de classificação das equações, quanto aos critérios de MDA, SD, SQRR, MDP, RQEM, V e r, utilizando-se o banco de dados 2 com a aplicação das equações estimadas pelo banco de dados 1 , por exemplo, foi atribuída a nota 1 para a equação que apresentou o melhor resultado de MDA, nota 2 à equação que se classificou em segundo lugar e, sucessivamente, até a equação de pior colocação. Repetiu-se este procedimento para todos os demais critérios estatísticos e englobou as notas em um único valor, denominado: Total de Notas (TN).

O procedimento anterior, feito no banco de dados 1 pela aplicação das equações estimadas pelo banco de dados 2, gerou também um valor TN para cada modelo. A soma dos dois valores de TN, para cada equação selecionada pela análise de resíduos com validação cruzada, gerou um valor geral denominado: Total Geral de Notas (TGN). Assim, baseando-se no menor valor de TGN e, também na análise gráfica da distribuição de resíduos, pôde-se classificar algumas equações de perfil do tronco para uso no plantio de $C$. citriodora amostrado.

\section{Resultados e Discussão}

Dentre as equações de perfil do tronco, geradas a partir dos 34 modelos estatísticos avaliados, preliminarmente, algumas foram descartadas por apresentarem insatisfatória distribuição de resíduos incluindo as equações geradas por modelos muito difundidos no Brasil, tais como: Baldwin Jr e Feduccia (1), Kozak et al. I (3 e 32), Ormerod I (6 e 33), Hradetzky (8 - com expoentes 1; 0,0005 e 6), Demaerschalk (2 e 31) e Schöefer II (34). Todos com EPR maior que 6,5\% e $R^{2}$ aj menor que 0,981 (Tabela 1 e Figura 1).

Ressalta-se que, dentre as versões testadas dos modelos de Kozak, Ormerod e Demaerschalk, o modelo 32 foi melhor que o 3 (Kozak), o modelo 6 foi melhor que o 33 (Ormerod) e o modelo 2 foi melhor que o 31 (Demaerschalk). Também, se observa na Tabela 1 que houve variação no EPR (4,20\% a 8,63\%) bem menor do que foi constatado em alguns estudos brasileiros que avaliaram estes modelos, onde houve variação de 3,93\% a 14,82\% (Andrade, 2014; Azevedo et al., 2017; Souza et al., 2018; Farias et al., 2019).

Por outro lado, complementarmente a análise inicial da distribuição de resíduos, as equações de perfil do tronco que mais se destacaram para C. citriodora foram comparadas considerando-se EPR $<5,5 \%$ e $\mathrm{R}^{2} \mathrm{aj}>0$,985. Como resultado, somente as equações advindas pelo ajuste dos modelos $4,5,7,13,15,17,22$, 23, 29 e 30 é que atenderam a esta condição de EPR e Rªj (Tabela 1). Além disso, ao se analisar a distribuição de resíduos destas dez equações (Figura 1), se observa que houve expressiva melhoria na distribuição de resíduos ocorrendo de forma mais equilibrada e homogênea ao longo do eixo de zero $\mathrm{cm}(0,0 \mathrm{~cm})$ que as equações $1,2,6,8,32$ e 34 . 
Tabela 1. Resultados das equações de perfil do tronco geradas a partir de 16 modelos estatísticos ajustados para C. citriodora.

\begin{tabular}{|c|c|c|c|c|c|c|c|c|}
\hline & \multicolumn{8}{|c|}{ Modelo Estatístico } \\
\hline & 1 & 2 & 4 & 5 & 6 & 7 & 8 & 13 \\
\hline$\beta 0$ & & 0,25160 & 1,40147 & 1,43250 & 1,05163 & 0,85387 & 126,13911 & \\
\hline$\beta 1$ & 2,07243 & 1,03179 & 0,77469 & 0,98458 & 0,81284 & 1,13454 & $-0,63604$ & $-0,06279$ \\
\hline$\beta 2$ & 0,29861 & 0,80267 & 1,01184 & $-0,14410$ & & $-3,32737$ & $-125,19749$ & 0,64449 \\
\hline$\beta 3$ & 77,13812 & $-1,00097$ & $-0,06738$ & 0,56632 & & 13,17724 & $-0,45164$ & \\
\hline$\beta 4$ & & & $-0,02447$ & $-0,76480$ & & $-30,04959$ & & \\
\hline$\beta 5$ & & & $-0,66046$ & 0,69334 & & 31,47030 & & \\
\hline$\beta 6$ & & & 0,48990 & 1,24464 & & $-12,66762$ & & \\
\hline$\beta 7$ & & & 0,16809 & 0,01110 & & & & \\
\hline$\beta 8$ & & & - & $-0,05322$ & & & & \\
\hline EPR & 6,53 & 6,52 & 4,44 & 4,20 & 6,56 & 4,69 & 6,54 & 4,87 \\
\hline \multirow[t]{3}{*}{$R^{2} a j$} & 0,980 & 0,980 & 0,991 & 0,992 & 0,980 & 0,990 & 0,980 & 0,989 \\
\hline & \multicolumn{8}{|c|}{ Modelo Estatístico } \\
\hline & 15 & 17 & 22 & 23 & 29 & 30 & 32 & 34 \\
\hline$\beta 0$ & & & 2,02168 & & & 1,07107 & 1,37962 & 1,22131 \\
\hline$\beta 1$ & 1,49574 & $-1,43628$ & 0,77506 & $-0,18468$ & 1,50132 & $-0,00391$ & $-2,71802$ & $-2,52704$ \\
\hline$\beta 2$ & 0,82213 & 11,43861 & 1,01223 & 3,27597 & 0,90449 & $-0,18476$ & 1,47729 & 6,50833 \\
\hline$\beta 3$ & 1,00821 & 0,71657 & $-1,00574$ & $-5,11088$ & 2,47579 & 0,49679 & & $-10,98418$ \\
\hline$\beta 4$ & 0,27529 & & 0,03569 & 4,03099 & $-3,34161$ & & & 8,83836 \\
\hline$\beta 5$ & $-0,01646$ & & $-2,13603$ & $-0,98144$ & 1,90630 & & & $-3,15885$ \\
\hline$\beta 6$ & $-0,02720$ & & 1,42729 & & & & & \\
\hline$\beta 7$ & 0,02039 & & 0,02564 & & & & & \\
\hline$\beta 8$ & 0,49166 & & & & & & & \\
\hline EPR & 4,75 & 4,36 & 4,51 & 5,13 & 4,93 & 5,48 & 8,63 & 6,59 \\
\hline$R^{2} a j$ & 0,990 & 0,991 & 0,991 & 0,988 & 0,989 & 0,986 & 0,966 & 0,980 \\
\hline
\end{tabular}

$\beta 0$ à $\beta 8=$ coeficientes estimados, $R^{2}$ aj=coeficiente de determinação ajustado, EPR=erro padrão residual em porcentagem.

Dentre as dez melhores equações, se observam que apenas duas foram obtidas a partir de modelos muito difundidos no Brasil, como o modelo 7, versão diferenciada do modelo de Schöepfer (1966), denominada de Schöefer I, e o modelo 13 de Guimarães e Leite (1992). Também, cinco equações se referem à modelos pouco testados no Brasil, quais sejam, modelos: 4 (Kozak, 1988), 5 (Kozak, 2004), 15 (Muhairwe, 1999), 17 (Andrade, 2014) e 22 Muhairwe (1993). Por fim, as três equações restantes, se referem a modelos que ainda não foram testadas no Brasil, quais sejam, modelos: 23 (Cervera, 1973), 29 (Lee et al., 2003) e 30 (Sharma \& Parton, 2009).

Na Tabela 1, ao se considerar apenas as dez equações de perfil do tronco que se sobressaíram, se observa que houve pouca variação de $\operatorname{EPR}(4,20 \%$ a $5,48 \%)$ e $R^{2}$ aj $(0,986$ a 0,992$)$. Neste caso, por serem altos valores de $R^{2}$ aj $(>0,985)$ e baixos valores de EPR (próximo de 3,67\%), não há base conclusiva para inferir sobre uma equação se sobressair a outra quanto ao uso em plantio com C. citriodora. 

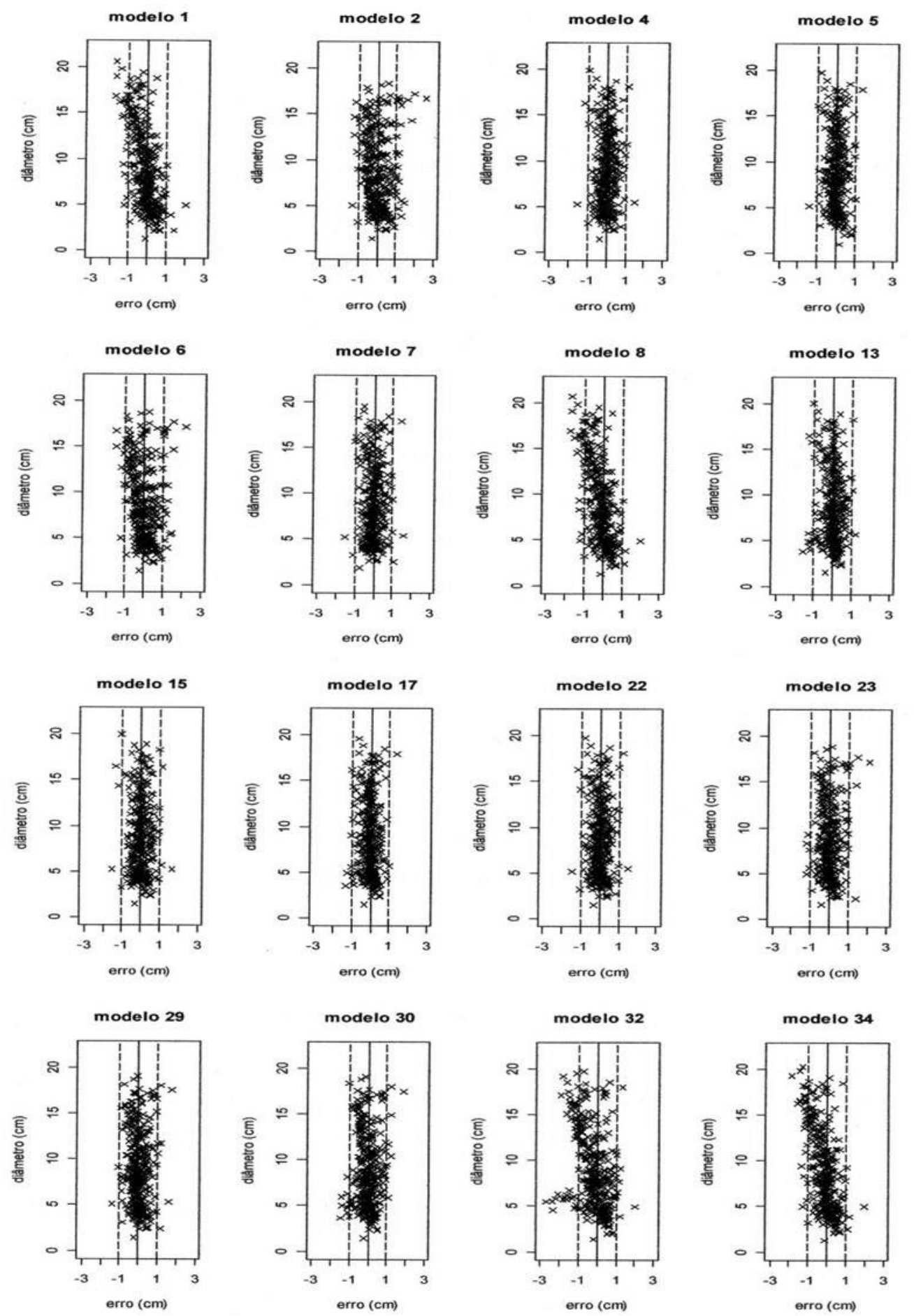

Figura 1. Erro em relação do diâmetro estimado obtido para 16 modelos de perfil do tronco de C. citriodora.

Por outro lado, em uma validação cruzada das dez equações de perfil do tronco previamente selecionadas, se observou pela análise da distribuição de resíduos que as equações advindas dos modelos 5 (Kozak, 2004), 13 (Guimarães \& Leite, 1992), 17 (Andrade, 2014) e 30 (Sharma \& Parton, 2009) apresentaram uma distribuição de resíduos bastante tendenciosa e, sendo assim, foram excluídas da análise seguinte.

Diante disso, considerando-se apenas as seis equações de perfil do tronco que mais se destacaram, advindas do ajuste dos modelos 4, 7, 15, 22, 23 e 29 (Figuras 2 e 3), se observa que os modelos: 4 (Kozak, 1988), 15 (Muhairwe, 1999) e 29 (Lee et al. 2003) se destacaram com respectivos valores de TGN iguais à 44, 45 e 40 pontos (Tabela 2). Dentre estes, o modelo 29 (Lee et al. 2003) se sobressaiu dos demais com TGN=40. 

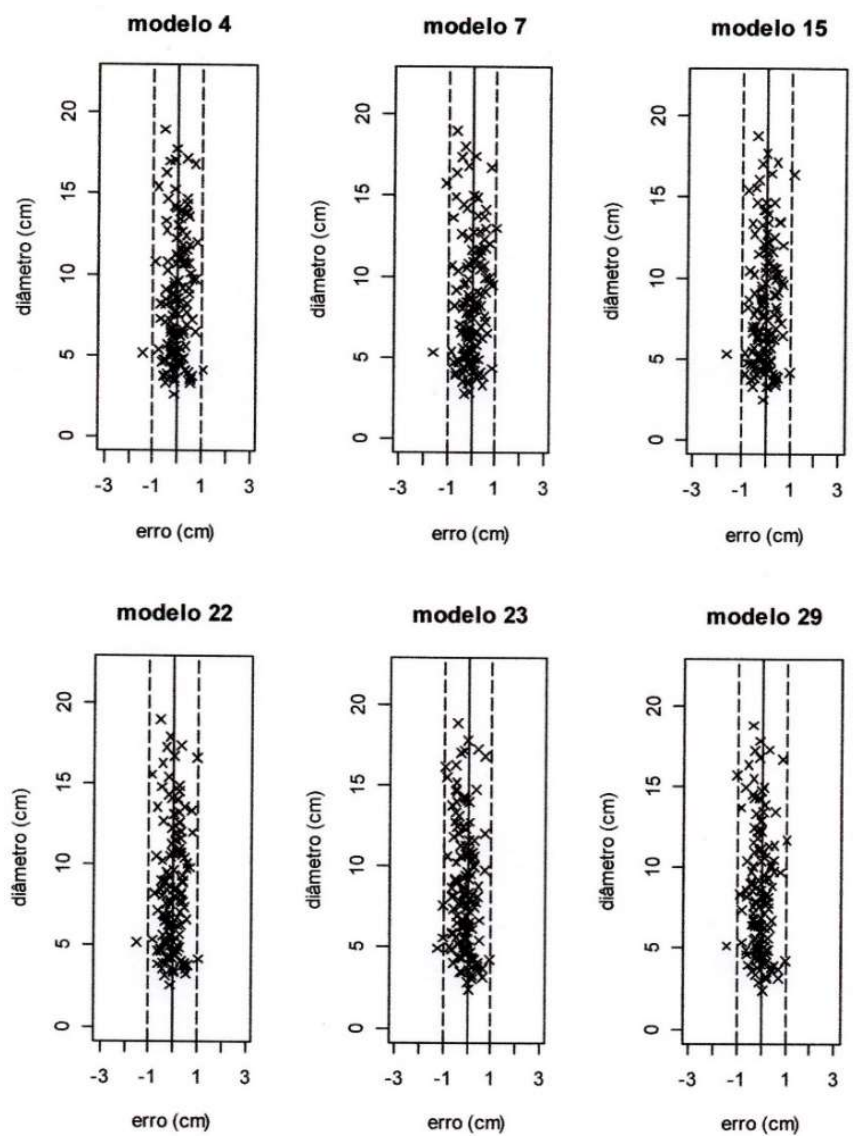

Figura 2. Erro em relação do diâmetro predito do tronco de C. citriodora obtido para seis modelos ajustados empregando-se o banco de dados 1 e aplicados no banco de dados 2 ( $n=132)$.

Apesar do menor valor de TGN ser obtido pela equação gerada a partir do modelo 29, optou-se por considerar a menor amplitude de TN (Tabela 2), que foi obtida pela equação estimada a partir do modelo 4 com 2 pontos (23-21=2) em comparação dos modelos $15(35-10=25)$ e $29(24-16=8)$. Portanto, pela menor amplitude de TN, a equação gerada a partir do modelo 4 (Kozak, 1988) demonstrou ser a mais estável para descrever o perfil do tronco de C. citriodora na área e região de amostragem. 

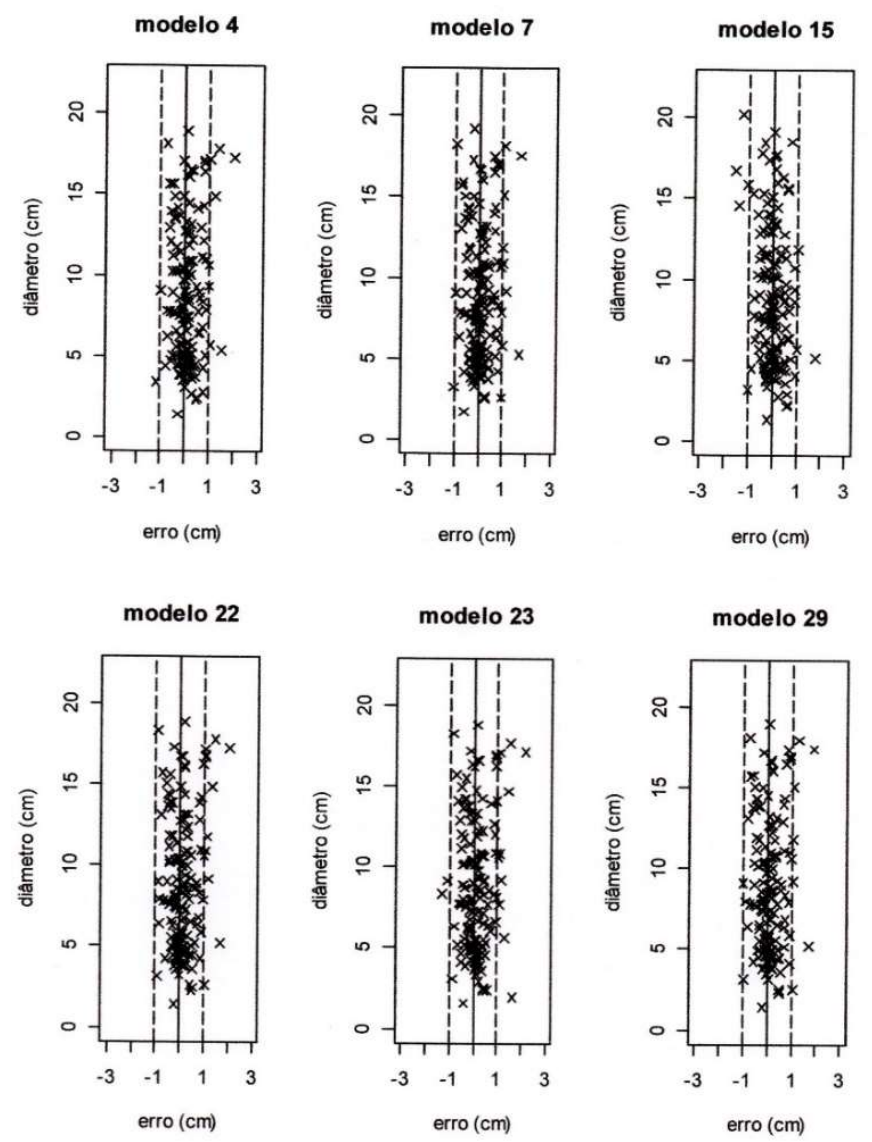

Figura 3. Erro em relação do diâmetro predito do tronco de C. citriodora obtido para seis modelos ajustados empregando-se o banco de dados 2 e aplicados no banco de dados 1 ( $n=151)$.

Tabela 2. Resultados do desempenho de seis equações de perfil do tronco geradas a partir do ajuste de seis modelos estatísticos para C. citriodora.

\begin{tabular}{|c|c|c|c|c|c|c|c|c|c|c|}
\hline ME & MDA & SD & SQRR & MDP & RQEM & VIÉS & DPD & ryx & TN & TGN \\
\hline \multicolumn{11}{|c|}{ Ajuste com banco de dados 1 e validação no banco de dados $2(0,2 ; 0,7 ; 4,7 ; 8,7 \ldots)$} \\
\hline 4 & $0,369^{2}$ & $0,493^{2}$ & $0,944^{4}$ & $0,88^{3}$ & $1,385^{4}$ & $0,112^{4}$ & $0,243^{2}$ & $0,993^{2}$ & 23 & 44 \\
\hline 7 & $0,373^{3}$ & $0,493^{2}$ & $1,040^{5}$ & $0,57^{2}$ & 1,2022 & 0,0972 & $0,243^{2}$ & $0,993^{2}$ & 20 & 50 \\
\hline 15 & $0,357^{1}$ & $0,482^{1}$ & $0,908^{3}$ & $0,24^{1}$ & $0,233^{1}$ & $0,019^{1}$ & $0,232^{1}$ & $0,994^{1}$ & 10 & 45 \\
\hline 22 & $0,392^{5}$ & $0,522^{4}$ & $0,857^{1}$ & $1,08^{5}$ & $1,544^{5}$ & $0,125^{5}$ & $0,272^{4}$ & $0,993^{2}$ & 31 & 52 \\
\hline 23 & $0,415^{6}$ & $0,545^{5}$ & $1,076^{6}$ & $1,06^{4}$ & $1,588^{6}$ & 0,1296 & 0,2975 & $0,992^{3}$ & 41 & 69 \\
\hline 29 & $0,380^{4}$ & $0,502^{3}$ & $0,873^{2}$ & $1,06^{4}$ & $1,329^{3}$ & $0,108^{3}$ & $0,252^{3}$ & $0,993^{2}$ & 24 & 40 \\
\hline \multicolumn{11}{|c|}{ Ajuste com banco de dados 2 e validação no banco de dados $1(0,4 ; 2,7 ; 6,7 \ldots)$} \\
\hline 4 & $0,319^{2}$ & $0,402^{4}$ & 0,6694 & $-1,04^{2}$ & $0,272^{2}$ & $-0,024^{2}$ & $0,162^{4}$ & 0,9951 & 21 & - \\
\hline 7 & 0,3495 & 0,4376 & 0,6845 & $-1,094$ & 0,1961 & $-0,0171$ & 0,1916 & 0,9942 & 30 & - \\
\hline 15 & $0,328^{4}$ & $0,418^{5}$ & $0,769^{6}$ & $-1,345$ & $0,430^{4}$ & $-0,037^{4}$ & $0,175^{5}$ & $0,994^{2}$ & 35 & - \\
\hline 22 & $0,319^{2}$ & $0,400^{3}$ & $0,648^{3}$ & $-1,05^{3}$ & $0,322^{3}$ & $-0,028^{3}$ & $0,160^{3}$ & $0,995^{1}$ & 21 & - \\
\hline 23 & $0,321^{3}$ & $0,394^{2}$ & $0,611^{2}$ & $-1,50^{6}$ & 1,3096 & $-0,114^{6}$ & $0,156^{2}$ & 0,9951 & 28 & - \\
\hline 29 & $0,303^{1}$ & $0,393^{1}$ & $0,583^{1}$ & $-0,991$ & $0,726^{5}$ & $-0,063^{5}$ & 0,1541 & $0,995^{1}$ & 16 & - \\
\hline
\end{tabular}

$\mathrm{ME}=$ modelos estatístico, MDA=média das diferenças absolutas, $\mathrm{SD}=$ desvio padrão das diferenças, $\mathrm{SQRR}=\mathrm{soma}$ de quadrado dos resíduos relativos, $M D P=$ média dos resíduos porcentuais, $R E Q M=r a i z$ quadrada do erro médio, $D P D=d e s v i o$ padrão das diferenças absolutas, ryx=coeficiente de correlação linear, TN=total das notas e TGN = total geral das notas para os dois bancos de dados. Valores em expoente são as notas de cada ME.

\section{Conclusões}

Seis equações se destacaram pelo bom desempenho, quais sejam advindas dos modelos: 4 (Kozak, 1988), 7 (Schöepfer, 1966 - modificado), 15 (Muhairwe I, 1999), 22 Muhairwe (1993), 23 (Cervera, 2003) e 29 (Lee et al. 2003), destacando-se os modelos de 7, 22, 23 e 29 que ainda não foram testados no país; 
As equações geradas a partir dos modelos de mais uso no Brasil (Demaerschalk, Kozak et al., Ormerod, Hradeztky e Schöefer), obtiveram desempenho muito insatisfatórios para descrever o de perfil do tronco de $C$. citriodora;

Dentre 34 equações geradas, as que foram advindas dos modelos: 4 (Kozak, 1988), 15 (Muhairwe I, 1999) e 29 (Lee et al. 2003), se sobressaíram na descrição do perfil do tronco de C. citriodora. Dentre estes, o modelo 4 (Kozak, 1988) demonstrou ser o mais estável.

\section{Agradecimentos}

À Coordenação de Aperfeiçoamento de Pessoal de Nível Superior (CAPES) pela bolsa de pesquisa.

\section{Referências}

Andrade, V.C.L. (2014). Modelos de taper do tipo expoente-forma para descrever o perfil do fuste de árvores. Pesquisa Florestal Brasileira, 34(80), 271-283. DOI: http://doi.org/10.4336/2014.pfb.34.80.614.

Andrade, V.C.L., Souza, A.L., Amaro, M.A., Costa, W.S., \& Soares, C.P.B. (2016). Perfil do fuste de Anadenanthera peregrina descrito pelo método da altura relativa. Ciência Florestal, 26(03), 875-887.

Azevedo, G.B.D., Azevedo, G.T.D.O.S., Castro, R.V.D.O., Barreto-Garcia, P.A.B., \& Rezende, A.V. (2017). Bole profile of eucalyptus under two conduction regimes in the southwest region of the Brazilian state Bahia. Revista Árvore, 41(02), 01-11. DOI: http://dx.doi.org/10.1590/1806-90882017000200011.

Batista, J.L.F. Couto, H.T.Z., \& Marquesini, M. (2001). Desempenho de modelos de relações hipsométricas: estudo em três tipos de floresta. Scientia Forestalis, 60, 143-163.

Cellini, J.M., Pastur, G.M., Wabö, E., \& Lencinas, M.V. (2002). Modelos de perfil de tronco en Nothofagus pumilio (Poepp. et Endl.) Krasser y su utilización en el cálculo del volumen total. Investigación Agraria: Sistemas y Recursos Forestales, 11(02), 246-261.

Cerqueira, C.L., Môra, R., Tonini, H., Arce, J.E., Carvalho, S.P.C., Ciarnoschi, L.D. (2018). Funções de afilamento para eucalipto em sistema de integração Lavoura-Pecuária-Floresta. Advances in Forestry Science, Cuiabá, 5(4), 479-485. DOI:http://doi.org/10.34062/afs.v5i4.7346.

Farias, K.J., Marcos, F.N., \& Natália, S.A. (2019). Quantificação do volume total por meio de modelos volumétricos e de afilamento em Eucalyptus grandis W. Hill ex Maiden. Revista de Ciências Agroveterinárias, 18(01), 33-40. DOI: http://doi.org/10.5965/223811711812019033.

Fischer, F., Scolforo, J.R.S., Acerbi, F.W., Mello, J.M., \& Maestri, R. (2001). Exatidão dos modelos polinomiais não segmentados e das razões entre volumes para representar o perfil do tronco de Pinus taeda. Ciência Florestal, 11(11), 167-188.

Instituto Nacional de Meteorologia [INMET] (2016). Disponível em: www.inmet.gov.br/portal/. Acesso em: 10/12/2019.

Lanssanova, L.R., Ubialli, J.A., Arce, J.E., Pelissari, A.L., Favalessa, C.C., Drescher, R. (2013). Avaliação de funções de afilamento para a estimativa de diâmetro de espécies florestais comerciais do bioma amazônico mato-grossense. Floresta, 43(02), 215-224.

Môra, R. (2015). Funções de afilamento de forma variável e modelagem de efeitos mistos em fustes de Pinus taeda e Eucalyptus saligna (Tese de Doutorado). Universidade Federal do Paraná, Curitiba, PR.

Muller, M.D., Salles, T.T., Paciullo, D.S.C., Brighenti, A.M., \& Castro, C.R.T. (2014). Equações de altura, volume e taper para eucalipto e acácia estabelecidos em sistema silvipastoril. Floresta, 44(03), 473-484.

Ribeiro, J.R., \& Andrade, V.C.L. (2016). Equações de Perfil do Tronco para Eucalyptus camaldulensis Dehnh no Centro-sul Tocantinense. Floram, 23(04), 534-543. DOI: http://dx.doi.org/10.1590/2179-8087.133215.

Schneider, P.R., Schneider, P.S.P., \& Souza, C.A.M. (2009). Análise de regressão aplicada à Engenharia Florestal. 3a ed. Santa Maria, RS: UFSM/CEPEF. 
Scolforo, J.R.S. (1993). Mensuração florestal 3: Relações quantitativas em volume, peso e a relação hipsométrica. Lavras, MG: ESAL/FAEPE.

Souza, G.S.A.D., Cosenza, D.N., Araújo, A.C.D.S.C., Pimenta, L.V.A., Souza, R.B., Almeida, F.M., Leite, H.G. (2018). Evaluation of non-linear taper equations for predicting the diameter of eucalyptus trees. Revista Árvore, 42(1), 1-10. DOI: http://dx.doi.org/10.1590/1806-90882018000100002. 